\title{
Preparation and application of highly porous aerogel-based bioactive materials in dentistry
}

\author{
Andrea KUTTOR ${ }^{1}$, Melinda SZALÓKI ${ }^{1}$, Tünde RENTE ${ }^{1}$, Farkas KERÉNYI ${ }^{1}$, József BAKÓ ${ }^{1}$, István FÁBIÁN ${ }^{2}$, \\ Attila JENEI ${ }^{3,4}$, István LÁZÁR ${ }^{2}$, and Csaba HEGEDS $(\varangle)^{1}$
}

1 Department of Prosthetic Dentistry and Biomaterials, Faculty of Dentistry, University of Debrecen, 4012 Debrecen, Hungary

2 Aerogel Research Group, Department of Inorganic and Analytical Chemistry, Faculty of Science, University of Debrecen, 4010 Debrecen, Hungary

3 Department of Dental Biochemistry, Faculty of Dentistry, University of Debrecen, 4012 Debrecen, Hungary 4 Department of Biophysics and Cell Biology, University of Debrecen, 4012 Debrecen, Hungary

(C) Higher Education Press and Springer-Verlag Berlin Heidelberg 2014

ABSTRACT: In this study, the possibility of preparation and application of highly porous silica aerogel-based bioactive materials are presented. The aerogel was combined with hydroxyapatite and $\beta$-tricalcium phosphate as bioactive and osteoinductive agents. The porosity of aerogels was in the mesoporous region with a maximum pore diameter of 7.4 and $12.7 \mathrm{~nm}$ for the composite materials. The newly developed bioactive materials were characterized by SEM. The in vitro biological effect of these modified surfaces was also tested on SAOS-2 osteogenic sarcoma cells by confocal laser scanning microscopy.

KEYWORDS: aerogel; sol-gel technique; bioactive material; SAOS-2 cell

\section{Contents}

1 Introduction

2 Materials and methods

2.1 Reagents

2.2 Synthesis of aerogels with bioactive modifications

2.3 Pore size analysis

2.4 Scanning electron microscopy (SEM)

2.5 Cell culture

2.6 Confocal laser scanning microscopy (CLSM)

3 Results and discussion

3.1 Synthesis of aerogels

3.2 Results of porosity measurements

3.3 Morphology of aerogel-based bioactive materials

Received February 18, 2014; accepted February 26, 2014

E-mail: chegedus@edu.unideb.hu
3.4 Cell attachment, confocal microscopy experiment 4 Conclusions

5 Abbreviations

References

\section{Introduction}

Recently, bone replacement has been an effective solution for the treatment of bone illnesses healing spontaneously very slowly or not healing at all. For the treatment of bone defects, various animal- or human-derived and artificial materials can be used. Over the past few years, remarkable progress has been achieved in the field of synthetically produced biomaterial [1]. Nowadays, in dentistry, osseoinductive and osseoconductive materials are widely used to fill alveolar bone defects, as implantable scaffolds to satisfy the customers' expectations [2-3].

Inorganic materials, mostly calcium phosphates [4] are 
1 used in bone surgery [5], which are in addition bioactive and osteoinductive, so they can be used as scaffolds. The stages that are involved in forming the bone bond of bioactive glasses and bioactive glass-ceramics were 5 summarized by Hench $[2,6]$. The surface characteristics of commercially available bioactive glasses and ceramics are usually modified [7]. Functional forms of these surfaces are often biologically active calcium phosphate layers, like hydroxyapatite (HA) which ensures the bonding interface

10 with tissues [8]. The HA layers are structurally and chemically identical with the mineral phase of bone and provides the interfacial bonding [9]. Various bioactive material types are used in orthopaedic surgery, such as 45S5 Bioglass $^{\circledR}, 58 \mathrm{~S}, 77 \mathrm{~S}$ glasses [10] or glass-ceramics in

15 different systems [11]. Metals with ceramic surface coatings can also be used [12].

Natural-based materials, including polysaccharides (chitin/chitosan, hyaluronic acid, alginate) or proteins (soy, collagen, fibrin gels) [8] may serve as a framework

20 for porogen materials, e.g. chitosan powder, which can be incorporated in bone cement aiming to improve its mechanical properties [13].

Recently, more and more sol-gel derived siliconsubstituted biomaterials came into focus of interest.

25 Silicon-substituted HA has been used in orthopedic, dental and maxillofacial surgery as a bone substitute. This bioactive material is an attractive and innovative solution for enhancing bone tissue growth rate, thereby improving early mechanical bone-fixation and thus leading to an

30 enhancement in the lifetime of implants [14]. The requirement for artificial bone substitute materials is the appropriate pore size [15-16].

The aim of the study was to prepare mesoporous silica containing biomaterials for dental application, using HA 35 and $\beta$-tricalcium phosphate ( $\beta$-TCP), as bioactive agents.

\section{Materials and methods}

\subsection{Reagents}

Tetramethoxysilane (Sigma-Aldrich, St. Louis, MO, USA), acetone, ammonia solution, methanol (Molar Chemicals, Budapest, Hungary), dried dimethyl sulfoxide (DMSO) (VWR, Debrecen, Hungary), microcrystalline cellulose for 45 column chromatography $(20-160 \mu \mathrm{m}$ in diameter, Merck, Darmstadt, Germany), $\beta$-TCP, HA and nanopowder HA $(<200 \mathrm{~nm}$ (BET, Sigma-Aldrich, St. Louis, USA)) were used as received. Water was triple deionized and carbon filtered. All chemicals were of reagent grade.

\subsection{Synthesis of aerogels with bioactive modifications}

For preparation of our samples two different solutions were prepared. The first solution (A), consisted of methanol solution $(10 \mathrm{~mL})$ of tetramethoxysilane (TMOS) $(3.00$ $\mathrm{mL}$ ). The second solution (B) consisted of methanol (10.8 $\mathrm{mL})$, dried DMSO $(1.2 \mathrm{~mL})$, water $(1.6 \mathrm{~mL})$, and aqueous ammonia solution $(7 \mathrm{~mol} / \mathrm{L}, 1.7 \mathrm{~mL})$ and microcrystalline cellulose $(1 \mathrm{~g})$. To solution $\mathrm{B}, \beta$-TCP $(1.00,0.25$, or $0 \mathrm{~g})$ and HA $(0,0.75$, or $1.00 \mathrm{~g})$ were added, respectively, and homogenized carefully. Solutions A and B were combined and homogenized again, then poured in plastic molds, where they solidified to alcogels in approximately $30 \mathrm{~min}$. The molds were made of poly(vinyl chloride) (PVC) tubes, and the bottoms were covered by glass slides. A thin layer of commercial silicon was sprayed onto the inner walls prior to use.

Alcogels were dried to aerogels in a custom-designed autoclave by using supercritical carbon dioxide at $80^{\circ} \mathrm{C}$. The samples were heat-treated in a furnace (Wise Therm FM-PH20, Daiham Sci. Co, Korea) with a temperature gradient of $500^{\circ} \mathrm{C}$ and $1000^{\circ} \mathrm{C}$ in $100^{\circ} \mathrm{C}$ increments. Approximately $1 \mathrm{~mm}$ thick discs were cut from the sintered monoliths with a serrated diamond hard tissue Leitz 1600 microtome (Ernst Leitz Wetzlar GmbH, Wetzlar, Germany).

\subsection{Pore size analysis}

The porosities of samples were characterized by nitrogen adsorption porosimeter $\left(\mathrm{NOVA}^{\circledR} 2200 \mathrm{e}\right.$, Quantachrome Instr., Boynton Beach, Florida, USA). Samples were measured out into a glass container (approx. $45 \mathrm{mg}$ ). The samples were vacuum degassed at $300^{\circ} \mathrm{C}$ for $3 \mathrm{~h}$ before the nitrogen gas sorption-desorption process.

\subsection{Scanning electron microscopy (SEM)}

SEM studies were performed by a Hitachi S-4300 instrument (SEM) equipped with a Bruker energy dispersive X-ray spectroscope (Hitachi Science Systems, Ltd., Japan). The surfaces of modified aerogels were covered by a sputtered gold conductive layer, and 5-10 kV accelerating voltage was used for taking high resolution electron micrographs. 


\subsection{Cell culture}

SAOS-2, malignant osteogenic sarcomas $\left(\right.$ ATCC $^{\circledR}$ HFB$85^{\mathrm{TM}}$, Rockville, MD, USA) were cultured in low glucose Dulbecco's modified Eagle's medium (DMEM), supplemented with $10 \%$ fetal bovine serum (FBS), $1 \%$ penicillinstreptomycin (Sigma-Aldrich, St. Louis, MO, USA), and 1\% GlutaMax (Gibco, Life Technologies, Grand Island, NY, USA). Cell cultures were maintained at $37^{\circ} \mathrm{C}$ under

\subsection{Confocal laser scanning microscopy (CLSM)}

The four-day cultured cells were fixed with aceton and stained using Alexa Fluor 488 phalloidin, and propidium iodide (PI) (Molecular Probes, Life Technologies, Grand Island, NY, USA). Cells were washed three times in phosphate buffered saline (PBS) buffer $(0.15 \mathrm{~mol} / \mathrm{L} \mathrm{NaCl}$, $3.2 \mathrm{mmol} / \mathrm{L} \mathrm{KCl}, 8.7 \mathrm{mmol} / \mathrm{L} \mathrm{Na}_{2} \mathrm{HPO}_{4} \times 12 \mathrm{H}_{2} \mathrm{O}, 1.7$ $\mathrm{mmol} / \mathrm{L} \mathrm{KH}_{2} \mathrm{PO}_{4}$ ) at $\mathrm{pH} 7.4$ and incubated with fluorescent dyes (5 unit/well) at room temperature for $30 \mathrm{~min}$ in the dark. Thereafter cells were washed three times in PBS and identified using Olympus FluoView-1000 laser scanning microscope (Olympus Imaging America Inc., Center Valley, PA, USA). Images were obtained of control, A and $\mathrm{C}$ samples.

\section{Results and discussion}

\subsection{Synthesis of aerogels}

Aerogel composite samples A (containing $1 \mathrm{~g} \beta$-TCP, $0 \mathrm{~g}$ HA), B (0.25 g $\beta$-TCP, $0.75 \mathrm{~g} \mathrm{HA})$ and $\mathrm{C}(0 \mathrm{~g} \beta$-TCP, $1 \mathrm{~g}$ HA) were received after supercritical drying of the corresponding alcogels (Fig. 1). The aim of the preparation of aerogel composites and nanocomposites by the sol-gel

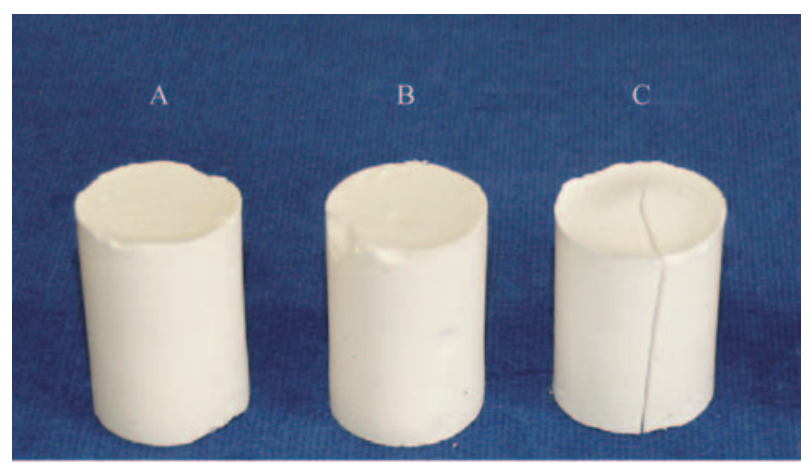

Fig. 1 Aerogel composites A (containing $1 \mathrm{~g} \beta$-TCP, $0 \mathrm{~g} \mathrm{HA}$ ); B $(0.25 \mathrm{~g} \beta$-TCP, $0.75 \mathrm{~g} \mathrm{HA})$ and $\mathrm{C}(0 \mathrm{~g} \beta$-TCP, $1 \mathrm{~g} \mathrm{HA})$ received after supercritical drying.

\subsection{Results of porosity measurements}

Specific surface areas and average pore diameters are presented in Table 1. The average pore diameters clearly indicate that these bioactive modified aerogels belong to the group of mesoporous materials [16]. Samples A and C showed similar specific surface areas and pore diameters. Sample B was significantly different in its physisorption properties from samples A and C. Sample B contained a mixture of $0.25 \mathrm{~g}$ of $\beta$-TCP and $0.75 \mathrm{~g}$ of HA, and has shrunk more intensively, which resulted in a lower specific surface area and average pore diameter due to the simultaneous embedding of both of $\beta$-TCP and HA bioactive materials. Sample $C$, which contained nanosized HA only, showed the highest porosity. As a consequence of its ability to be uniformly distributed in the matrix, in contrast to micron-sized inorganic fillers, this may form macroscopic inhomogeneities.

\subsection{Morphology of aerogel-based bioactive materials}

SEM images of modified aerogels are presented in Fig. 3. Sample A proved to be more vulnerable to mechanical stress than samples B and C. Its structure was damaged more than that of the others, because of the lower surface adhesion and less reinforcing effect of high-melting point TCP crystals compared to either micron- or nano-sized HA particles. Aggregation of $\beta$-TCP grains can be observed inside the holes. Samples B and C showed more compact structure on the SEM picture; both of them contained hydroxyapatite, which developed stronger adhesion with the matrix. Sample C contained nano-sized HA distributed homogenously in the matrix, without forming a separated phase. The micron-sized inorganic fillers HA and $\beta$-TCP in sample B formed separated phases inside the matrix. 

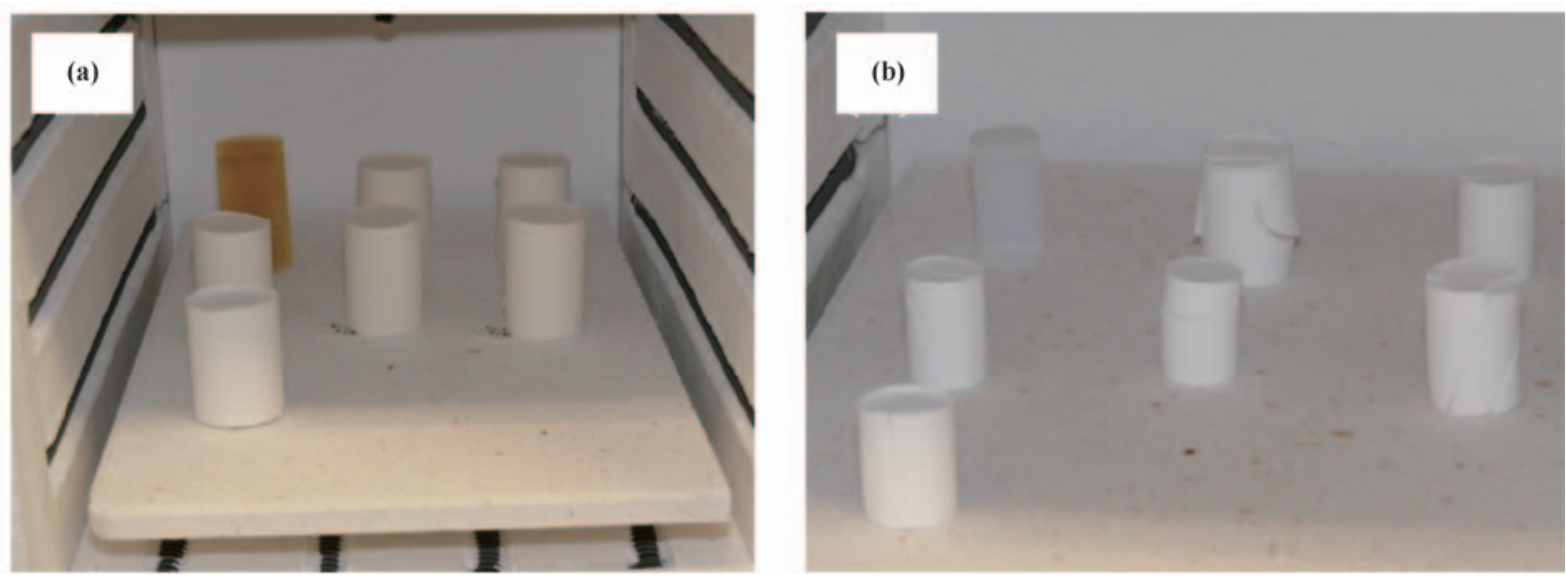

Fig. 2 Standard aerogel and modified aerogels (a) before and (b) after heat-treatment. Left: $25^{\circ} \mathrm{C}$; right: $1000^{\circ} \mathrm{C}$. The yellowish sample in picture (a) is the basic aerogel, and the others are bioactive modified aerogels.

Table 1 Surface area (BET) and average pore diameter $(\mathrm{BJH})$ data of bioactive modified aerogels $\left(R^{2}\right.$ is the linear regression coefficient of BET determination)

\begin{tabular}{lccc}
\hline Sample & BET $/\left(\mathrm{m}^{2} \cdot \mathrm{g}^{-1}\right)$ & $\mathrm{BJH} / \mathrm{nm}$ & $R^{2}$ \\
\hline A & 118 & 12.7 & 0.9999 \\
B & 78 & 7.4 & 0.9997 \\
C & 125 & 12.7 & 0.9998 \\
\hline
\end{tabular}

However, HA reacted with silica matrix on the grain 25 boundaries leading to a lower melting region, and it resulted in a higher degree of shrinking on heating at higher temperatures. The complete embedding of the nano-HA particles prevented autonomous thermal behaviours at the phase borders, and the reinforced nano-composite behaved more like a homogeneous aerogel monolith. It resulted in an increased mechanical strength with the preservation of high porosity. The elemental composition of the modified aerogels was confirmed by X-ray fluorescence elemental analyses.

\subsection{Cell attachment, confocal microscopy experiment}

The prerequisite for successful osseointegration of the implant in vivo is the attachment of stem cells/precursor

40 cells to the implant surface (reviewed in Ref. [17]). In order to demonstrate cellular activities including cell spreading and proliferation of SAOS-2, malignant osteosarcoma cell line was used as our model system. The cells plated and cultured on coverslip (control) and modified aerogels 45 samples $\mathrm{A}$ and $\mathrm{C}$. We could not investigate the cell behaviour on sample B because it broke into small pieces during slicing. Visualization of the cytoskeleton (phalloidin) and nucleus (propidium iodide) by confocal laser scanning microscopy demonstrated that the osteosarcomas are spreading, and remained as coherent cells (Fig. 4). The behaviour of the osteosarcomas on modified surfaces is very similar to that observed on coverslip, however, on sample C, there were areas not covered by cells. These "not-covered-areas" look specific for sample C. These areas could be due to the differences of the charging and/or the surface structure between the two samples. However, further experiments are needed to clarify this observation and to clear up the reasons.

\section{Conclusions}

In this study, aerogel composite samples $\mathrm{A}$ (containing $1 \mathrm{~g}$ $\beta$-TCP, $0 \mathrm{~g} \mathrm{HA}), \mathrm{B}(0.25 \mathrm{~g} \beta$-TCP, $0.75 \mathrm{~g} \mathrm{HA})$ and $\mathrm{C}(0 \mathrm{~g} \beta$ TCP, $1 \mathrm{~g}$ HA) were prepared and examined by cell attachment, porosity and scanning measurements. Sample $\mathrm{C}$, which contained nano-sized HA only, showed the highest porosity. As a consequence of the nanoparticles' ability to be uniformly distributed in the matrix, composite $\mathrm{C}$ showed the lowest thermal shrinking and good mechanical strength, in contrast to other micron-sized inorganic fillers, which may form agglomerates in the matrix. Sample A proved to be more vulnerable to 

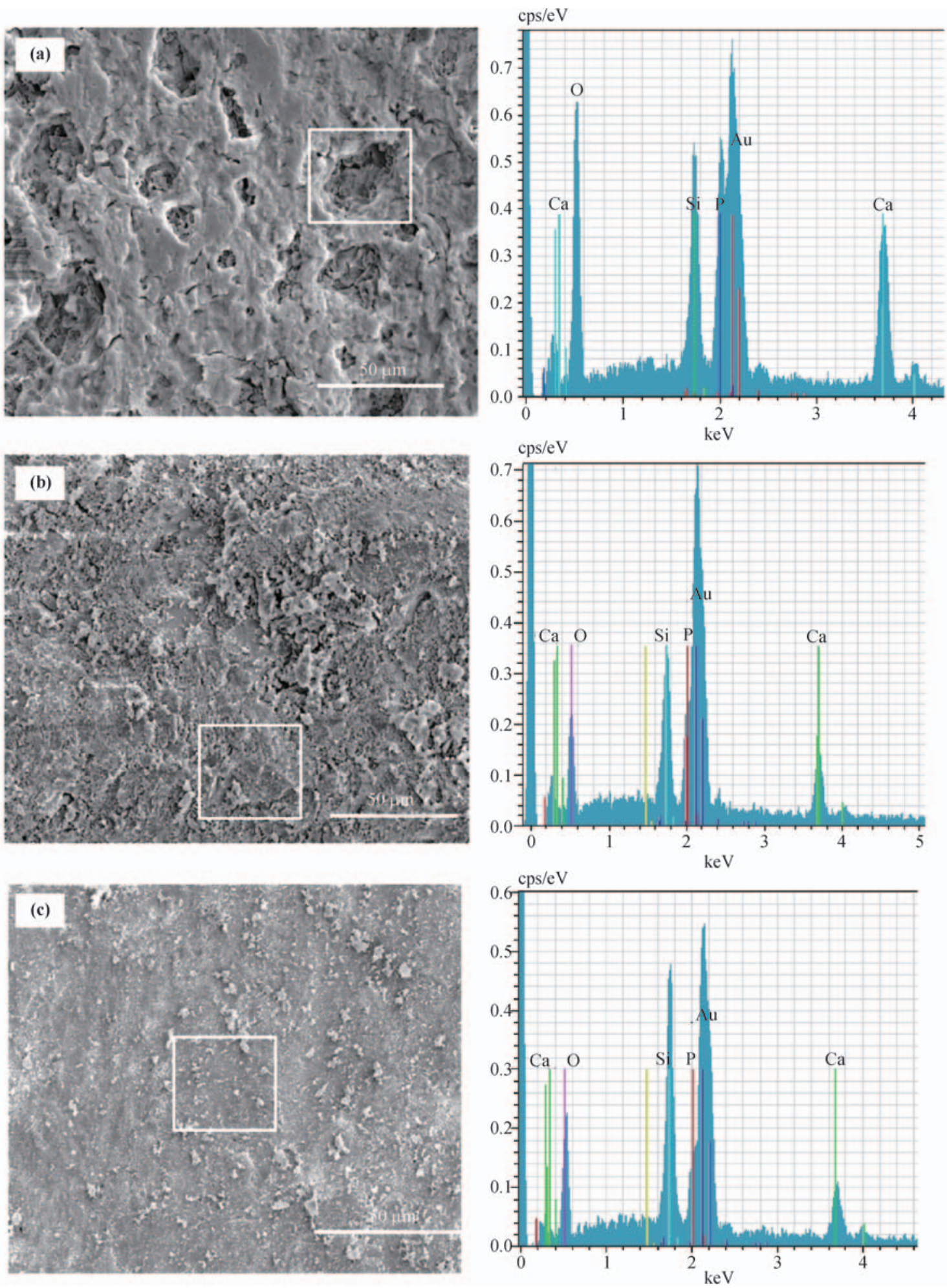

Fig. 3 SEM images and X-ray fluorescence spectra of the surface of aerogel-based bioactive materials: (a) sample A; (b) sample B; (c) sample C (bar: $50 \mu \mathrm{m})$. 


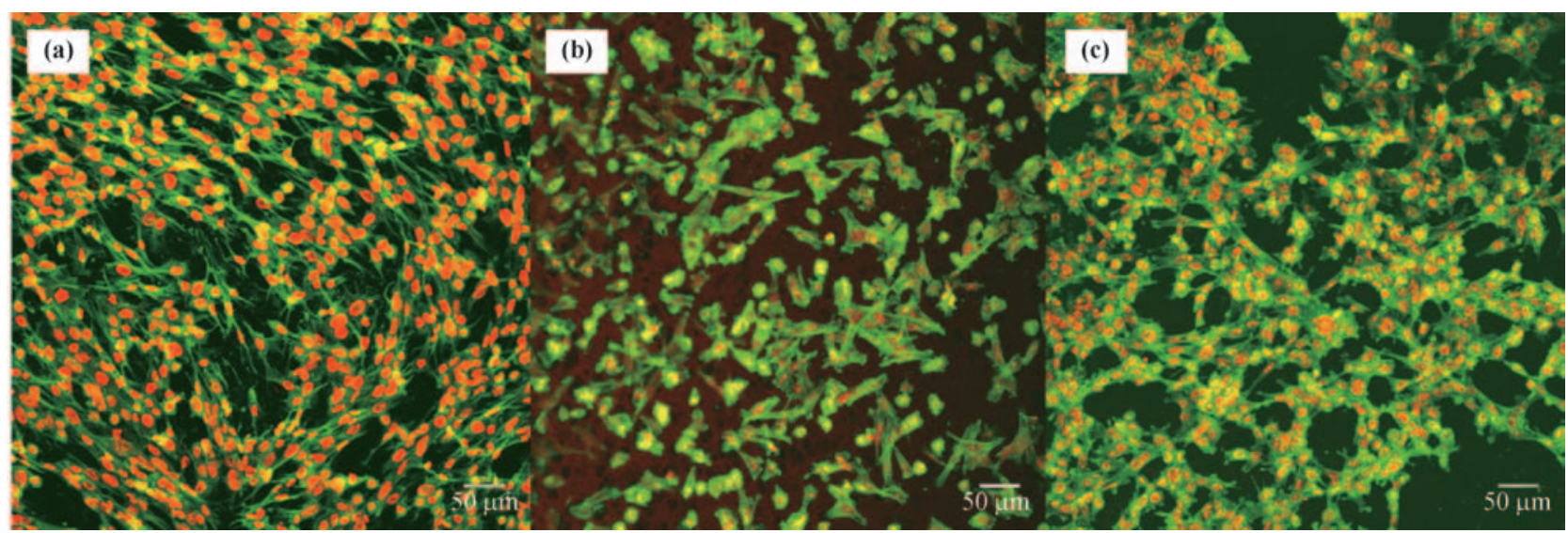

Fig. 4 Confocal images of SAOS-2 cells plated on (a) coverslip (control), (b) sample A and (c) sample C aerogels. The cells were

mechanical stress than samples B and C. The latter one presented more compact structure on the SEM picture; both $\mathrm{B}$ and $\mathrm{C}$ contained HA, which developed strong adhesion with the matrix. SAOS-2 cells were plated and cultured on 20 glass (control) and modified aerogel samples A and C. The behaviour of the osteosarcomas on modified aerogels was very similar to that observed on glass slide. Based on these measurements, we have demonstrated that these aerogel composite samples are biocompatible and non-toxic for this cell type, so it might find practical applications in the dental field in the future.

\section{Abbreviations}

$\beta$-TCP beta-tricalcium phosphate

BET Brunauer-Emmett-Teller method

BJH Barrett-Joyner-Halenda method

CLSM confocal laser scanning microscopy

35 DMEM Dulbecco's modified Eagle's medium

DMSO dimethyl sulfoxide

FBS fetal bovine serum

HA hydroxyapatite

PBS phosphate buffered saline

PI propidium iodide

40 PVC poly(vinyl chloride)

SEM scanning electron microscopy

TMOS tetramethoxysilane

45

Acknowledgements The work/publication was supported by the TÁMOP-4.2.2.A-11/1/KONV-2012-0036 project and OTKA76834. The project was co-financed by the European Union and the European Social Fund.

\section{References}

[1] Lázár I, Manó S, Jónás Z, et al. Mesoporous silica-calcium phosphate composites for experimental bone substitution. Biomechanica Hungarica, 2010, III.1: 151-158

[2] Dorozhkin S V. Calcium orthophosphates in dentistry. Journal of Materials Science: Materials in Medicine, 2013, 24(6): 1335-1363

[3] Dorozhkin S V. Calcium orthophosphate cements for biomedical application. Journal of Materials Science, 2008, 43(9): 30283057

[4] Dorozhkin S V. Biphasic, triphasic and multiphasic calcium orthophosphates. Acta Biomaterialia, 2012, 8(3): 963-977

[5] Monchau F, Hivart P, Genesite B, et al. Calcite as a bone substitute. Comparsion with hydroxyapatite and tricalcium phosphate with regard to the osteoblastic activity. Materials Science and Engineering C, 2013, 33(1): 490-498

[6] Hench L L. Bioceramics. Journal of the American Ceramic Society, 1998, 81(7): 1705-1728

[7] Gittings J P, Bowen C R, Dent A C, et al. Electrical characterization of hydroxyapatite-based bioceramics. Acta Biomaterialia, 2009, 5(2): 743-754

[8] Rezwan K, Chen Q Z, Blaker J J, et al. Biodegradable and bioactive porous polymer/inorganic composite scaffolds for bone tissue engineering. Biomaterials, 2006, 27(18): 3413-3431

[9] Hench L L. Bioceramics: from concept to clinic. Journal of the American Ceramic Society, 1991, 74(7): 1487-1510

[10] Silver I A, Deas J, Erecińska M. Interactions of bioactive glasses with osteoblasts in vitro: effects of $45 \mathrm{~S} 5$ Bioglass ${ }^{\circledR}$, and $58 \mathrm{~S}$ and $77 \mathrm{~S}$ bioactive glasses on metabolism, intracellular ion concentrations and cell viability. Biomaterials, 2001, 22(2): 175-185

[11] Ohtsuki C, Kamitakahara M, Miyazaki T. Bioactive ceramicbased materials with designed reactivity for bone tissue regeneration. Journal of the Royal Society, Interface, 2009, 6(Suppl 3): 

S349-S360

[12] Liu X, Morra M, Carpi A, et al. Bioactive calcium silicate ceramics and coatings. Biomedicine \& Pharmacotherapy, 2008, 62 (8): 526-529

[13] Lee E J, Shin D S, Kim H E, et al. Membrane of hybrid chitosansilica xerogel for guided bone regeneration. Biomaterials, 2009, 30(5): 743-750

[14] Balamurugan A, Rebelo A H S, Lemos A F, et al. Suitability evaluation of sol-gel derived Si-substituted hydroxyapatite for dental and maxillofacial applications through in vitro osteoblasts response. Dental Materials, 2008, 24(10): 1374-1380

[15] Karageorgiou V, Kaplan D. Porosity of 3D biomaterial scaffolds and osteogenesis. Biomaterials, 2005, 26(27): 5474-5491

[16] Sing K S V, Everett D H, Haul R A W, et al. Reporting physisorption data for gas/solid systems with special reference to the determination of surface area and porosity. Pure and Applied Chemistry, 1985, 57(4): 603-619

[17] Tuan R S. Role of adult stem/progenitor cells in osseointegration and implant loosening. International Journal of Oral \& Maxillofacial Implants, 2011, 26(Suppl): 50-62, discussion 63-69 